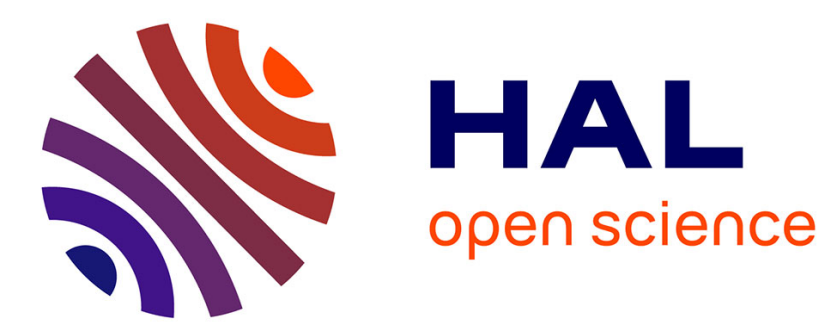

\title{
Le don et l'initiation: de l'impact de la littérature sur les cultes de possession au Brésil \\ Véronique Boyer
}

\section{To cite this version:}

Véronique Boyer. Le don et l'initiation: de l'impact de la littérature sur les cultes de possession au Brésil. L'Homme - Revue française d'anthropologie, 1996, 138, pp.7-24. hal-01415777

\section{HAL Id: hal-01415777 \\ https://hal.science/hal-01415777}

Submitted on 13 Dec 2016

HAL is a multi-disciplinary open access archive for the deposit and dissemination of scientific research documents, whether they are published or not. The documents may come from teaching and research institutions in France or abroad, or from public or private research centers.
L'archive ouverte pluridisciplinaire HAL, est destinée au dépôt et à la diffusion de documents scientifiques de niveau recherche, publiés ou non, émanant des établissements d'enseignement et de recherche français ou étrangers, des laboratoires publics ou privés. 


\title{
Persée
}

http://www.persee.fr

\section{Le don et l'initiation. De l'impact de la littérature sur les cultes de possession au Brésil}

\author{
Véronique Boyer \\ L'Homme, Année 1996, Volume 36, Numéro 138 \\ p. $7-24$
}

Voir l'article en ligne

\section{Avertissement}

L'éditeur du site "PERSEE » - le Ministère de la jeunesse, de l'éducation nationale et de la recherche, Direction de l'enseignement supérieur, Sous-direction des bibliothèques et de la documentation - détient la propriété intellectuelle et les droits d'exploitation. A ce titre il est titulaire des droits d'auteur et du droit sui generis du producteur de bases de données sur ce site conformément à la loi n`98-536 du 1 er juillet 1998 relative aux bases de données.

Les oeuvres reproduites sur le site «PERSEE » sont protégées par les dispositions générales du Code de la propriété intellectuelle.

Droits et devoirs des utilisateurs

Pour un usage strictement privé, la simple reproduction du contenu de ce site est libre.

Pour un usage scientifique ou pédagogique, à des fins de recherches, d'enseignement ou de communication excluant toute exploitation commerciale, la reproduction et la communication au public du contenu de ce site sont autorisées, sous réserve que celles-ci servent d'illustration, ne soient pas substantielles et ne soient pas expressément limitées (plans ou photographies). La mention Le Ministère de la jeunesse, de l'éducation nationale et de la recherche, Direction de l'enseignement supérieur, Sous-direction des bibliothèques et de la documentation sur chaque reproduction tirée du site est obligatoire ainsi que le nom de la revue et- lorsqu'ils sont indiqués - le nom de l'auteur et la référence du document reproduit.

Toute autre reproduction ou communication au public, intégrale ou substantielle du contenu de ce site, par quelque procédé que ce soit, de l'éditeur original de l'oeuvre, de l'auteur et de ses ayants droit.

La reproduction et l'exploitation des photographies et des plans, y compris à des fins commerciales, doivent être autorisés par l'éditeur du site, Le Ministère de la jeunesse, de l'éducation nationale et de la recherche, Direction de l'enseignement supérieur, Sous-direction des bibliothèques et de la documentation (voir http://www.sup.adc.education.fr/bib/ ). La source et les crédits devront toujours être mentionnés. 


\section{Le don et l'initiation}

De l'impact de la littérature sur les cultes de possession au Brésil

Véronique BoYER, Le don et l'initiation. De l'impact de la littérature sur les cultes de possession au Brésil. - Dans les cultes de possession au Brésil, les médiums opposent le don et l'initiation. On tente de comprendre cette opposition en considérant les incidences des écrits, scientifiques et ésotériques, sur la constitution actuelle du champ religieux. Les premiers, en s'appuyant sur les paradigmes de la connaissance savante, ont en effet défini des normes rituelles que maintiendrait un système initiatique. Les seconds en revanche ont échoué dans leur entreprise de systématisation, encourageant l'expression de versions personnelles inspirées par les esprits. La formation d'une « tradition » religieuse ne peut être saisie qu'en tenant compte des rapports ambivalents entre écriture et oralité.

A

u Brésil, les cultes de possession ont longtemps été sévèrement réprimés par les autorités pour «exercice illégal de la médecine », « abus de confiance » et « idolâtrie». Au cours du XIX siècle, nombreuses furent les arrestations de chefs de culte et les destructions d'objets cultuels (certains, sauvés du désastre, ont été réunis à Rio de Janeiro dans le musée de la police militaire où on peut encore les voir aujourd'hui).

Ces attaques virulentes ont suscité, à partir des années 1930, deux réactions reposant pareillement sur l'écrit mais résolument distinctes en ce qui concerne leur point d'ancrage géographique, l'origine sociale des auteurs et le propos de leur répartie. D'une part, une ouvre scientifique qui émane de membres des élites intellectuelles et s'adresse dans un premier temps à elles prend la défense des cultes de Bahia, capitale de l'un des États de la région Nord-Est. D'autre part, à Rio de Janeiro et São Paulo, centres urbains du SudEst, est publiée une littérature ésotérique rédigée par des proches des cultes, appartenant à la petite classe moyenne, à l'intention de fidèles peu lettrés et peu fortunés.

Si la répression est aujourd'hui terminée, le statut des cultes de possession reste ambigu. Ils sont tout à la fois stigmatisés (les épithètes appliquées aux 
médiums sont sévères 1 , marginalisés (les terreiros, lieux de culte, situés dans les quartiers périphériques ne sont pas d'un accès facile), très fréquentés (il n'est pas rare d'y rencontrer les membres de la classe moyenne, voire des élites) et contribuent au même titre que le carnaval ou le football à l'affirmation d'une identité nationale (la fête en l'honneur de Iemanjá, divinité des eaux salées, est l'occasion de réjouissances publiques dans le pays entier). Le regard porté par la société brésilienne sur les cultes de possession et leurs fidèles est ainsi pour le moins équivoque.

Or la ligne médiane passant entre ce qui est acceptable des cultes de possession et ce qui l'est moins vient recouper celle qui sépare les représentations spécifiques qu'en ont données les textes savants et les écrits occultistes : la religion des orixás et la magie des esprits, ou caboclos, sont deux facettes des cultes simultanément explorées et fortement contrastées. On trouve une résonance de ces élaborations dans les terreiros avec les discussions incessantes et passionnées des médiums autour de la transmission du savoir, alors même que tous offrent dans l'action religieuse des services semblables aux consultants : l'accès à travers les possédés aux conseils et à l'aide d'êtres invisibles pour soulager les malheurs des hommes.

Les médiums ou «fils-de-saint » reconnaissent certes que la compétence d'un spécialiste religieux est fonction de son talent à exploiter un ensemble de connaissances, formant un corpus rituel et doctrinaire auquel ses pairs se réfèrent également. Cependant ils tentent aussi inlassablement de distinguer ce qui relève de l'initiation par un maître de ce qui est du ressort du «don», des capacités personnelles que le médium manifeste lors de son apprentissage. $\mathrm{Si}$ les fils-de-saint s'interrogent sur les chances (matérielles) de l'un ou de l'autre de se faire initier, ils répètent par ailleurs à l'envi que cela n'est pas suffisant, voire nécessaire, pour être un grand médium. Chacun d'eux semble en fait s'évertuer à briser la complémentarité de l'initiation et du don dans l'acquisition de savoir pour établir la prééminence de l'un des deux termes sur l'autre ${ }^{2}$. Certains assurent de leur attachement à ce qui est de l'ordre de l'aptitude personnelle et innée tandis que d'autres se prononcent en faveur d'une initiation en bonne et due forme.

Le contraste entre ces notions se nourrit de la divergence entre les intérêts de chefs de culte soucieux de consolider leur ascendant sur des médiums qu'ils doivent former et les aspirations de ces derniers désireux de fonder un centre qui leur appartienne. Car l'inflation de l'exigence d'une initiation pour soi et pour les autres est en étroite relation avec le succès de la carrière religieuse des fils-de-saint. De ce point de vue, la récurrence du motif dans l'expression des conflits incite à se pencher sur les rapports de pouvoir qu'il permet de construire à l'intérieur de l'univers religieux en tenant compte d'un environnement sociologique plus large: l'ensemble de la société brésilienne. Les posi-

1. Voir sur ce thème, le travail d'Anaíza Vergolino (1976).

2. Je remercie Mme Carmen Bernand d'avoir attiré mon attention sur cette spécificité des cultes afro-brésiliens. 
tions des chefs de culte se définissent en effet par rapport aux sources écrites évoquées précédemment, investies d'une autorité et d'un prestige que tentent de s'approprier les maîtres des terreiros. Le don et l'initiation apparaîtront alors moins liés par une opposition réelle, reconduite à différents niveaux et dans différents contextes, que par un effet de polarisation permettant d'organiser un vaste champ sémantique où d'autres éléments s'inséreront.

\section{Les terreiros entre don et initiation}

À Belém³ ${ }^{3}$ les médiums - en majorité des femmes issues des milieux pauvres - insistent sur leur proximité avec les caboclos $^{4}$ qui les possèdent lors des cérémonies. Un "voyage initiatique au fond de l'eau " confirmerait souvent une élection que laissaient déjà présager maintes visions pendant l'enfance et l'adolescence. Durant ce voyage unique en son genre, qui n'est pas sans évoquer le récit chamanique, les caboclos dispenseraient aux fils-de-saint les fondements d'un savoir indispensable à l'accomplissement de la mission dont ils sont investis : s'abandonner à la possession afin que les esprits deviennent accessibles aux hommes souffrants. L'absence d'intermédiaire entre les médiums et les êtres invisibles dans leur formation s'expliquerait par le "don de naissance », celui qu'ils ont eu de tout temps et que nul ne peut contester. Le caboclo, par sa seule présence lors de la possession, rappellera cette élection initiale.

L'esprit qui les a guidés, ou un autre, deviendra un double s'immisçant sans vergogne dans l'intimité de leur maisonnée. À plus forte raison dans l'enceinte rituelle, les caboclos détenant résolument le pouvoir décideraient de tout, de la solution des problèmes que les consultants leur soumettent aux altérations du rituel. Dans ce cadre, l'habileté et la compétence du fils-de-saint lui sont données lors de la possession par les nombreux caboclos qu'il est capable de représenter. Ces prestations, qui attestent de la force du lieu unissant le possédé aux êtres invisibles, sont à la base de la différenciation des positions dans le terreiro et de la formation d'une hiérarchie implicite entre les médiums.

Quand le personnage de l'esprit est parfaitement associé au fils-de-saint, qu'il est donc reconnu par son entourage comme une partie de lui-même - ce dont témoigne par exemple la demande expresse des consultants de s'adresser à son caboclo - , tout médium cherche à ouvrir son propre terreiro où il sera à son tour père ou mère-de-saint, à la fois chef du groupe religieux et propriétaire du lieu de culte. Au regard de la position conférée aux esprits, le maître du

3. Ville de plus d'un million d'habitants située sur le delta de l'Amazone et capitale de l'État du Pará, appartenant à la région Nord du Brésil.

4. J'emploie ici le terme utilisé par les médiums. Les caboclos ont en fait une nature multiple : ils sont des esprits de morts, mais aussi de disparus (ils sont dit alors « enchantés », différence qu'établirait l'absence de décomposition đu corps) et encore d'animaux. Un même caboclo peut se manifester sous l'une de ces formes dans le lieu de culte. L'esprit peut enfin se présenter en tant qu'exu, mais en ce cas il change de nom. 
centre qu'il fréquente peu difficilement s'opposer à ce projet d'émancipation caressé par tous les fils-de-saint. Car un chef de culte est contraint d'accepter pour les autres ce dont il se réclame - le charisme de ses caboclos - posant alors, dans l'exercice du pouvoir, des limites étroites qui menacent la survie du terreiro qu'il a fondé. Quel que soit leur statut dans le terreiro, les participants aux cultes de possession s'abritent derrière les esprits qu'ils incarnent : le père ou la mère-de-saint pour diriger, les fils-de-saint pour le quitter.

Afin de contrer la prolifération d'autorités dans le groupe, les médiumscaboclos avec lesquels le maître (ou la maîtresse) d'un centre doit composer, ce dernier s'appuie sur les rituels mis à sa disposition par le système religieux. Ces cérémonies confirmant les progrès des fils-de-saint sont un instrument de contrôle d'autant plus efficace pour sceller son ascendant que leur préparation est laissée à la discrétion du chef de culte. Lors du baptême (batismo), dit aussi cruzamento, qui sanctionne une première étape dans l'apprentissage des médiums, le père ou la mère-de-saint consacre le fils-de-saint en lui versant sur la tête une préparation à base de feuilles macérées. Il est suivi, quand le maître du terreiro juge le moment opportun, du bori demandant un effort financier accru au fils-de-saint, en raison des sacrifices d'animaux qu'il comporte.

Enfin certains chefs de culte organisent l'initiation longue, complexe et très coûteuse ${ }^{5}$ qu'est la feitura do santo, quand un orixá, l'un des dieux venus d'Afrique et non plus un esprit-caboclo national, exige qu'un médium le serve. Au cours d'une période de réclusion d'une à trois semaines, le fils-de-saint, dont le corps est rasé, apprend les « fondements » de son orixá (ses pas de danse, les mets qu'il affectionne...) et reçoit sur le haut du crâne le sang des animaux à deux et quatre pattes consacrés à la divinité. Pendant la phase de claustration, l'initié est par trois fois présenté au public : tout d'abord il sort vêtu et peint de blanc, couleur d'Oxalá le créateur ; puis il apparaît habillé des couleurs du dieu qui l'a élu, lequel donnera à cette occasion son nom par la bouche du possédé : enfin, il se montre paré du costume et des ornements propres à son orixá. La période d'apprentissage se prolongera jusqu'à ce que le fils se soit acquitté des sept offrandes annuelles en l'honneur de l'orixá-électeur.

Il est cependant tout à fait remarquable qu'à Belém de nombreux propriétaires de terreiros, en fait la majorité jusqu'à la dernière décennie, semblent répugner à user de ces éléments initiatiques. Ainsi, rarement accompli à un moment distinct des rituels ordinaires, le baptême est généralement associé par les pères et mères-de-saint au «bain de tête » (banho de cabeça) dispensé lors d'une quelconque cérémonie de «développement» (desenvolvimento) pour renforcer le contact du médium avec ses caboclos. Les chefs de culte ne sont pas plus pressés d'organiser le bori qui n'établit pas de lien permanent dans le

5. En 1988 , le coût moyen d'un bori était d'environ 800 francs, celui de la première étape de la feitura do santo (la période de réclusion jusqu'à la troisième sortie) était de près de 2000 francs, sommes considérables (même si l'on tient compte des probables exagérations des fils-de-saint pour montrer leur dévouement aux orixás et caboclos) au regard des revenus des médiums, de un à trois salaires minimums par mois (300 à 900 francs). 
temps et l'espace avec leur personne dans leur terreiro. Ils redoutent que la cérémonie passée les fils-de-saint forts de cette nouvelle qualification religieuse s'éclipsent encore plus rapidement que les autres médiums comptant sur leurs seuls caboclos.

Quant à la feitura do santo, la plupart des maîtres de terreiro, qui l'ont rarement suivie eux-mêmes ${ }^{6}$, reculent devant les critiques que ne manquerait pas de susciter un rituel conduit par un initiateur non initié. Or les chefs de culte qui ont assis depuis toujours leur position sur la réputation de leur caboclos ne peuvent pas solliciter un père ou une mère « fait dans le saint » sans reconnaître l'imperfection de leur savoir et les limites du pouvoir des êtres invisibles qui les habitent puisque avec la feitura do santo, les esprits-caboclos se soumettraient aux dieux-orixás. Ce faisant, ils « effaceraient » en quelque sorte leurs longues années de pratique religieuse.

On voit donc les médiums osciller entre deux attitudes selon qu'ils sont ou non initiés. Certains s'appuient avant tout sur leur prestation de possédé dans le lieu de culte et sur les témoignages de leur proches pour soutenir leur notoriété. Assurant ne pas être pleinement convaincu du caractère fondamental de l'initiation, ils considèrent qu'un chef de culte offre aux fils-de-saint une connaissance " extrinsèque », tenue pour périphérique au savoir lui-même dépendant tout entier de l'expérience de la possession. Tout un chacun pourrait selon eux apprendre par une observation attentive tant les gestes à accomplir pour s'occuper du lieu de culte que les chants, les prières, les offrandes et les pas de danse appropriés ou la conduite correcte d'un rituel. En revanche, ceux qui ont subi une feitura do santo, ou même un bori, tendent à asseoir davantage leur renommée sur le prestige de leur filiation (la réputation du chef de culte qui les a initiés, et celle du père ou de la mère-de-saint qui a formé celui-ci) et sur l'étendue de leur savoir à l'état conscient, c'est-à-dire hors possession, dont ils donnent la mesure lors des discussions avec les autres médiums, «faits dans le saint » ou non.

Sur ce hiatus entre deux conceptions de l'acquisition de savoir est toutefois posée une passerelle confortée par le sentiment général «qu'il faut être compétent en tout, que ce soit en matière d'orixás ou de caboclos». Dès lors, chacun interprète à sa guise et selon son intérêt le rapport entre don et initiation : l'un affirme la vertu cardinale du «don de naissance », en reconnaissant qu'il peut être cultivé et renforcé par l'initiation ; l'autre soutient que la feitura do santo doit venir l'encadrer et le confirmer.

L'engrenage sans fin dans lequel sont pris ces deux termes - l'un ramenant toujours à l'autre - et l'énergie dépensée par les protagonistes, pour apparemment le rompre et plus sûrement le renforcer, incitent à s'interroger sur la nature de la tension entre don et initiation en tant qu'ils sont signifiants de catégories plus amples. Car ces catégories, permettant de lire les changements que l'on peut constater dans les pratiques cultuelles, renvoient d'abord à une

6. Ce sont surtout de jeunes médiums ou mères-de-saint qui optent pour cette solution. 
partition du champ religieux qui relève en partie de la littérature consacrée aux cultes, dans la mesure où elle a aidé à sa construction en l'alimentant d'autres mots.

\section{L'œuvre des intellectuels}

L'attention des élites intellectuelles pour les études afro-brésiliennes, éveillée dès la fin du siècle dernier, redouble dans les années 1930 avec les travaux de l'anthropologue Arthur Ramos et de ses disciples, entre autres Edison Carneiro et Gonçalves Fernandes. À l'initiative du sociologue Gilberto Freyre le premier congrès afro-brésilien est organisé en 1934 dans le plus grand théâtre de Recife, ville du Nord-Est du pays. Chacun des participants, qui sont venus de toutes les régions du Brésil, apporte dans sa discipline une contribution à la connaissance des noirs au Brésil : ethnologues, folkloristse, musicologues, linguistes, historiens, médecins psychiatres et neurologues, spécialistes en anthropologie physique et écrivains vont ainsi se succéder ${ }^{7}$. L'apport culinaire est également souligné par des chefs de culte qui livrent quelques recettes de « délices afro-brésiliens » au public érudit.

Trois ans plus tard a lieu dans la capitale de l'État voisin de Bahia un second congrès 8 qui gagne une dimension internationale grâce à l'appui de nombreuses personnalités (entre autres citons Melville Herskovits, Fernando Otiz, Robert Park, Percy Martin et Donald Pierson). Aux représentants des disciplines déjà mentionnées se joignent un peintre et le directeur de l'Institut Nina Rodrigues 9 qui organise une exposition d'objets cultuels.

Les pères et mères-de-saint, dont pas moins de quarante si l'on en croit Edison Carneiro (1964 : 98-10110) ont promis d'envoyer des délégations, participent très activement aux réunions : certains prestigieux chefs de culte présentent des communications et l'éminent devin Martiniano do Bonfim, principal informateur de Nina Rodrigues, est invité à présider la séance inaugurale. Ils éclairent également les distingués érudits et les membres des élites inscrits aux conférences sur les choses des cultes en les invitant à se rendre dans les terreiros de Bahia (ceci contrairement au congrès précédent où les évolutions des chefs de culte étaient soumises à l'appréciation de l'assemblée des savants au lieu même du colloque, sur la scène du théâtre Santa Isabel).

7. Mon propos n'est évidemment pas de discuter la valeur scientifique des analyses présentées. Ces interventions m'intéressent ici en tant que matériaux ethnogaphiques au même titre que la littérature umbandiste présentée plus loin.

8. Les actes de ces congrès ont été publiés : Estudos afro-brasileiros (Rio de Janeiro, Ariel Editora, 1935) réunit les communications de Recife ; O negro no Brasil (Rio de Janeiro, Civilizaçao Brasileira, 1940) rassemble celles de Bahia.

9. Raymundo Nina Rodrigues, pionnier des études afro-brésiliennes, mène ses enquêtes à la fin du siècle dernier.

10. Ce texte est initialement écrit en 1940 pour la publication des annales du congrès de 1937 mais j'utilise la réédition de 1964. Notons que Edison Carneiro est l'un des rares ethnologues à s'être par la suite élevé contre «la saison des spectacles du nègre » inaugurée par ces colloques dans un texte datant de 1953 (1964: 115). 
Cette illustration des thèmes du « folklore nègre » $11 \mathrm{a}$ eu, toujours selon Edison Carneiro (ibid. : 100), l'avantage d'en terminer « avec l'épouvantail qu'étaient encore, pour les classes dites supérieures de Bahia, les candomblés », les lieux de culte de la ville.

Il sera enfin décidé que les terreiros doivent se doter d'une existence jurisique. La commission exécutive se chargera donc à l'issue du congrès de créer un organisme qui réunira les chefs de la ville et de l'État : le 3 août 1937 l'Union des Sectes afro-brésiliennes de Bahia est fondée.

Sociologues et anthropologues de toutes nationalités (Brésiliens, Américains, Français) sont à l'avant-garde de cette entreprise de réhabilitation du noir et de sa culture qui dépassera largement les frontières du monde scientifique 12 . La description minutieuse de certains cultes de possession constitue l'essentiel de l'œuvre de ceux qui se sont joints à l' "école bahianaise », du nom de la ville où s'est déroulé le second congrès. En effet, le candomblé de Bahia que ces chercheurs se sont attachés à observer leur semble un exemple édifiant de la continuité entre l'Afrique et le Brésil.

Les débats se situent dans l'espace bien précis des "survivances", des « réinterprétations » et des particularités des «traditions » africaines représentées au Brésil. Aucune réelle différence de point de vue ne vient troubler des analyses indiscutablement convergentes, sinon uniformes, dans leurs conclusions qui affirment toutes la supériorité d'une version du candomblé, le culte Nagô. Les rares désaccords portent sur le choix des informateurs dont la fiabilité serait non seulement déterminée par leur position dans la hiérarchie religieuse mais aussi par leurs pratiques rituelles. Parce que les chercheurs entendent rendre justice à la grandeur de l'héritage africain, ils écarteront de la matière de leurs études les terreiros dits syncrétiques.

Les ethnologues recueillent auprès des chefs de culte formant un cercle relativement restreint les mythes concernant les orixás, les dieux venus d'Afrique, qui retracent leurs aventures, expliquent leurs liens de parenté, racontent leurs amitiés et leurs inimitiés, dépeignent leurs caractéristiques et leurs attributs. Ces mythes constituent la trame du ballet joué par les possédés, où chaque orixá est identifié par le costume et les ornements endossés par les fils-de-saint : le rituel, répétition fidèle (même si elle est incomplète) de ce que les anciens exécutaient, est ici le principal support de la mémoire collective. À ce titre, le thème de l'initiation n'est pas négligé. Sa longueur et la complexité de ses étapes sont soulignées, son rôle fondamental dans l'intégration de l'individu au groupe est relevé, tout comme est signalée l'ampleur des connaissances que doivent assimiler les postulants avant de pouvoir être habités par leur orixá.

11. Outre les cérémonies religieuses, les congressistes furent conviés à un spectacle de samba et à la démonstration d'une lutte appelée capoeira.

12. Dans les années 1960 , des peintres et sculpteurs comme Carybé, des romanciers comme Jorge Amado trouveront dans les cultes de possession une précieuse source d'inspiration. Comme l'écrit Reginaldo Prandi (1991 : 73) : « Tout mène à Bahia : le Cinéma Nouveau, les arts scéniques », la cuisine, la musique (qui présente les vénérables mères-de-saint de Bahia et fait connaître les noms des saints)...» 
La parfaite cohésion et la richesse du système symbolique et rituel sont ainsi montrées par ces analyses.

Une limite est pourtant fixée à ces dissertations où le commentaire laisse percer une profonde sympathie à l'endroit des gens des terreiros : l'interdit de divulguer ce qui se transmet dans la chambre initiatique (camarinha), de consigner dans leurs ouvrages ce qui constitue le « secret » des cultes. Ce refus est tout à l'honneur de ces anthropologues attentifs à ne pas trahir la confiance que leur ont accordée les pères et mères-de-saint. Cependant, parce qu'en même temps ils insistent fortement sur le caractère fondamental des connaissances confiées par le chef de culte aux initiés 13 , ce qui était au départ une question d'éthique se transformera, comme nous le verrons, en un problème d'orthodoxie qui aura des répercussions sur le devenir des cultes de possession.

\section{La littérature ésotérique}

À côté de cette production scientifique et à peu près à la même époque (puisque les premiers livres paraissent dans les années 1940) fleurit une seconde littérature, publiée par des maisons d'édition spécialisées, qui provient d'autres segments sociaux. En effet dans le Sud-Est du pays, des hommes issus de la petite classe moyenne, qui se réclament au moins dans un premier temps d'un courant - l'umbanda - différent du candomblé, semblent déterminés à entreprendre une "codification » (le terme est d'ailleurs contenu dans la plupart des titres de ces ouvrages) des cultes de possession auxquels ils participent, et ce une fois pour toutes, donc par écrit ${ }^{14}$.

Si ces auteurs sont animés par un souci analogue à celui qu'on perçoit sous la plume des scientifiques préoccupés de la réputation des cultes de possession, ils ne parlent plus d'Afrique et de tradition mais se placent d'entrée de jeu sous le signe de la modernité 15 et professent le blanchiment ${ }^{16}$. Exhortant les fidèles à purger le système religieux de ce qui est nègre, ils insistent sur la nécessité d'en finir avec les rites et les croyances pouvant évoquer l'Afrique (surtout les sacrifices d'animaux). L'Afrique noire, loin d'être conçue comme un lieu d'origine, aurait été une phase transitoire, et néfaste, dans l'histoire séculaire de cultes de possession venus d'Inde ou même d'Atlantide.

L'uniformisation souhaitée a pour ces umbandistes un double aspect : défi-

13. Pour une analyse du « secret», voir ZEMPLÉNI 1976.

14. Une partie importante de cette littérature est aussi composée de simples livres de recettes ou d'incantations, proche en cela des manuels d'occultisme européens utilisés par les voyants guérisseurs dans toutes les Antilles ( $c f$. Bougerol 1993).

15. La modernité est ici associée au spiritisme kardeciste qui, introduit au Brésil à la fin du siècle dernier, connut un franc succès auprès des classes dominantes. Si les ouvrages de son fondateur Allan Kardec ne sont pas mentionnés, leur auteur (de son vrai nom Léon Rivail) est, quant à lui, fréquemment cité. La doctrine de Kardec, fondée sur l'évolution à travers la réincarnation, s'accordait fort bien avec le positivisme d'Auguste Comte qui avait la faveur des partisans de la République. La devise du pays, "ordre et progrès », est d'ailleurs inspirée de l'œuvre de ce dernier.

16. Voir le livre de Renato Ortiz sur ce mouvement de blanchiment qu'exprime bien le titre de son livre : La mort blanche du sorcier noir (1978). 
nir les normes rituelles qui doivent encadrer les cérémonies et classer les esprits qui se manifestent dans les terreiros (caboclos et exus ici conçus comme des esprits de morts). En outre, afin de contrer le développement « anarchique » des lieux de culte, ils affirment que tout nouveau médium doit se placer sous la direction d'un père-de-saint le temps d'apprendre à contrôler la transe. Mais le terme « développement » (desenvolvimento), restreint dans son usage à l'expérience même de la possession, est préféré à celui d'initiation. Tenu pour une voie complémentaire dans l'apprentissage religieux, le livre prend une dimension importante comme source de savoir et les umbandistes fournissent souvent des bibliographies à leurs lecteurs.

Ils recommandent enfin aux chefs de culte de se regrouper au sein de fédérations. La première d'entre elles, l'Union spirite de l'Umbanda du Brésil, est constituée en 1939, soit deux ans après la naissance de l'Union des Sectes afrobrésiliennes réunissant les candomblés de Bahia. La distinction entre candomblé et umbanda, évidente pour les anthropologues, est cependant moins claire aux yeux des écrivains liés aux cultes de possession. Dès les années 1950, un courant de la littérature ésotérique s'écarte des tenants de l'umbanda blanche en proposant une forme africanisée du culte et crée une organisation concurrente. Courte rivalité qui s'éteindra, en 1956, avec la fondation d'une nouvelle fédération associant des partisans des deux bords, et auxquels se joindront par la suite des représentants du candomblé17. Dans le même temps, les auteurs umbandistes fondent des journaux, parviennent à se faire entendre sur les ondes et à la télévision; certains se lancent dans la politique et deux d'entre eux sont élus députés 18 .

Les interprétations du système religieux présentées dans ces ouvrages ne reposent pas sur des descriptions rigoureuses ou sur de savantes démonstrations. Elles se fondent sur la certitude de rapporter des messages révélés par les esprits. L'importance accordée aux dialogues des hommes, souvent les auteurs eux-mêmes, avec les êtres invisibles lors de visions ou par le truchement d'un possédé est rendue dans l'écriture par une profusion de majuscules, de guillemets et d'italiques. Des reproductions de peintures, dont la légende nous dit qu'elles sont faites par des médiums sous l'inspiration des esprits-caboclos, illustrent le texte là où les dessins du célèbre Carybé ou d'un autre artiste ornaient les ouvrages scientifiques.

Le projet de codification est alors limité par la reconnaissance de la toutepuissance des esprits. En effet, si la vérité n'est pas détenue par les êtres humains, aucun des auteurs ne peut contredire les idées qu'avancent les uns et les autres et se poser en tant qu'arbitre incontesté. Plus encore, ils sont obligés

17. Les fédérations naissent de la volonté des gens des cultes de se présenter en un ensemble religieux cohérent et disparaissent à la suite d'un conflit de pouvoir entre leurs dirigeants qui en créent alors de nouvelles. L'histoire des fédérations se caractérise par une tentative, incessante et systématiquement vouée à l'échec, de réunir dans une organisation unique et nationale les chefs de culte. Sur l'historique des fédérations, voir le travail de Diana Brown (1974).

18. Aux chambres de l'État de Rio de Janeiro (à l'époque Guanabara) et du Rio Grande do Sul, et non au Congrès fédéral. 
d'accorder aux chefs de culte la liberté d'adapter leurs directives en fonction des orientations « spirituelles » que leur communiqueraient leurs caboclos. Les variations sont en fait au principe même du culte comme le laisse entendre l'un d'entre eux qui écrit : "L'umbanda est universelle et ses formes multiples. » Sous la direction des « guides » invisibles sont donc développées dans les livres des conceptions hautement personnelles de l'umbanda.

Quelle que soit la position des auteurs fidèles des cultes, ils ne demeurent pas insensibles aux écrits des scientifiques. Nombre d'entre eux reprennent les classifications des cultes élaborées par les érudits, se réfèrent à l'œuvre des anthropologues, et semblent accepter les prémisses des études savantes afrobrésiliennes qui ont longtemps attribué à l'origine bantoue de l'umbanda son ouverture aux influences extérieures et son syncrétisme avancé. Freitas et Pinto (1970 : 69), par exemple, reconnaissent la suprématie nagô démontrée par les autorités scientifiques tout en affirmant que les «croyants bantous » sont en mesure de compenser ce handicap initial par l'application studieuse. D'autres adoptent apparemment une attitude plus radicale. Dans un ouvrage collectif intitulé Umbanda, religião do Brasil (1960: 133), un auteur dénie aux anthropologues les compétences pour pénétrer «les ressorts profonds » des cultes afro-brésiliens parce qu'ils « se basent sur la partie exotérique des cérémonies ». En fait, ce point de vue reflète l'attitude généralement adoptée par les littérateurs afro-brésiliens. Ils connaissent assez bien les interprétations savantes mais n'utilisent que les éléments compatibles avec leur propre conception des cultes. Dans un ouvrage récent, Diamantino F. Trindade (1991) fait ainsi un résumé de l'histoire des fédérations manifestement à partir du travail de Diana Brown (1974) (qu'il cite d'ailleurs dans sa bibliographie) en passant sous silence sa principale conclusion, à savoir que ces institutions renforcent le clientélisme.

Que ce soit face à leur coreligionnaires ou face aux scientifiques, ces écrivains adoptent une conduite semblable basée sur une logique de conciliation. Des littérateurs qui divergent sur presque tout (la classification des esprits, l'origine de l'umbanda, le déroulement du rituel) n'hésitent pas à se faire mutuellement référence sans pour autant expliquer ce qui les rapproche. $L^{\prime}$ ' ignorance » des divergences apparaît ici comme l'exigence de la constitution d'une globalité. Car les conceptions particularisantes de chacun où néanmoins rien ne semble contraire au culte n'empêchent pas, comme dans toute littérature ésotérique, de prétendre à la généralisation: c'est l'ampleur d'un mouvement, et non pas sa substance, que les citations réciproques tentent d'évoquer.

\section{L'africanisation des cultes}

Les ambitions de ces littératures qui ont toutes deux pris pour objet les cultes de possession sont fort différentes. Les auteurs afro-brésiliens, par leur 
entreprise de systématisation, cherchent à jeter les bases d'un droit de contrôle et d'un pouvoir de censure sur les terreiros. L'intention des ethnologues est bien évidemment tout autre. C'est l'intérêt scientifique, l'approfondissement de la connaissance des systèmes religieux, qui justifient en soi des études dont l'objectivité est revendiquée. Cependant l'œuvre de ceux qui prétendent jeter un regard impartial sur les cultes de possession aura paradoxalement des répercussions plus visibles sur les terreiros que la littérature ésotérique.

Il n'est ici pas vain de rappeler que ce qui se pense, se dit et s'écrit sur des cultes de possession est largement diffusé auprès de l'ensemble de la population. Les congrès et les manifestations publiques, appuyées par les structures institutionnelles tôt mises en place (les fédérations chargées de réunir les lieux de culte), sont abondamment commentées par la presse, la radio, la télévision...

Les gens des terreiros n'ignorent donc pas l'opinion des anthropologues qui dès le début témoignent de leur vive admiration pour le candomblé de Bahia respectueux des vieilles traditions africaines. Celui-ci est implicitement opposé à l'umbanda ancrée dans la société contemporaine et rejetée dans le mélange corrupteur et l'ignorance. Le dédain pour le syncrétisme, conséquence d'une percée des valeurs du monde moderne dans les lieux de culte, est manifeste. De la vulgarisation de la pensée des scientifiques, les fils-de-saint retiendront également que les orixás sont plus nobles que les caboclos et que l'initiation, si essentielle, dans le candomblé, est le seul moyen d'accéder véritablement au « secret», ce savoir occulte sur lequel un chef de culte fonde sa réputation. L'umbanda, exclue presque totalement des études savantes, se voit ainsi implicitement dénier la légitimité à laquelle elle aspire.

Or on observe dans toutes les régions du Brésil un mouvement d' " africanisation » des cultes de possession 19 . Un nombre croissant de médiums qui ont commencé leur itinéraire par l'umbanda rejoignent le candomblé ; des pères et mères-de-saint se font initier à ce culte et abandonnent leur «nation » d'origine 20 ; des chefs de culte introduisent dans leurs terreiros d'umbanda des éléments rituels et vestimentaires associés au candomblée 21.

Ce mouvement d'africanisation est d'autant plus intéressant qu'il ne se traduit pas par une stricte observance des règles du candomblé. La distinction entre candomblé et umbanda tranche en fait dans le vif d'une réalité mouvante, où les pratiques et croyances s'enchevêtrent au point de rendre ardue et épi-

19. On peut d'ailleurs penser que le projet de faire de l'umbanda la seule authentique religion brésilienne a échoué en raison de l'option africaniste des scientifiques, et plus généralement des élites intellectuelles. En effet, au Venezuela, c'est sous l'impulsion des classes dominantes et des artistes que le culte de Maria Lionza est devenu un symbole national (BARRETo RAMOs 1994).

20. J'ai fait état de cette tendance pour la ville de Belém (Boyer-ArAúJo 1993a). Reginaldo Prandi (1991 : 107-108) note également que le candomblé, arrivé à São Paulo dans les années 1950 , conquiert chaque jour davantage de fideles. Entre 1960 et 1980, « la trajectoire est, ou a été, la suivante : umbanda, angola, ketu ». Le candomblé ketu, originaire de Bahia et plus prestigieux que le culte angola, est ainsi au point d'arrivée de la plupart des parcours religieux.

21. Par exemple les contra-eguns, les bracelets de paille tressée que les médiums portent pour éviter la possession ; l'adjá, une cloche de métal qui est jointe aux tambours; ou l'aleá, le grand dais sous lequel sont conduits les possédés à l'occasion des fêtes publiques. 
neuse toute tentative d'établir des coupures nettes 22 . Candomblé et umbanda y forment davantage des pôles, des tendances organisant l'univers religieux que des courants opposés et inconciliables.

Depuis la fin des années 1970, des travaux ont renouvelé l'étude des cultes afro-brésiliens en montrant le rôle joué par les intellectuels dans la constitution du champ religieux ${ }^{23}$. Les ethnologues, qui ont concentré leurs recherches dans quelques-uns des nombreux terreiros de Bahia ${ }^{24}$, n'ont pas en effet perçu la flexibilité de la notion de tradition à laquelle en appelaient les pères et mèresde-saint pour démarquer leurs positions respectives. En traitant sur un mode érudit la revendication de racines africaines, ils ont en quelque sorte « folklorisé » les cultes de possession et gommé certains aspects du culte comme les caboclos : l'éradication de la magie était nécessaire à la consécration de la religion. Ils ont alors donné à la tradition un contenu et une forme explicites, contribuant de cette façon à la définition d'une orthodoxie qui dissocie le "vrai » d'un « faux », l'« authentique » de l'« apocryphe ». En d'autres termes, la tradition dans sa version actuelle est le produit d'une relation dialectique entre scientifiques et certains maitres de terreiro, les premiers attestant de l'intégrité des seconds, qui à leur tour confirment que ceux-ci ne sont pas fourvoyés.

Les retombées de ce processus sont telles que Reginaldo Prandi (1991 : 29) n'hésite pas à affirmer que dans le mouvement de « reconstruction religieuse du candomblé [à São Paulo] une bonne partie de la littérature [ethnographique] a pris la place de la transmission orale de l'ancien culte». Plus encore, dans les terreiros, " africaniser signifie aussi l'intellectualisation, l'accès à une littérature contenant les oracles d'Ifá, la réorganisation du culte selon les modèles ou éléments amenés d'Afrique contemporaine » (ibid. : 117). La banalisation surprenante du terme syncrétisme parmi les fidèles des cultes et la récurrence de la métaphore de l'initiation comme université (le don propre de l'umbanda étant alors l'apprentissage de base, l' «école primaire ») en disent par ailleurs long sur la fascination exercée par les intellectuels sur les médiums.

C'est dans ce contexte où le pouvoir du livre est reconnu que les pères-desaint s'essaient à l'écriture avec plus ou moins de succès auprès des éditeurs. Un médium, informateur d'Yvonne Maggie ${ }^{25}$ et un temps son étudiant (il abandonnera l'université peu après), publiera ainsi un livre où il retrace son itinéraire et expose ses idées au sujet des cultes. L'écrit s'impose dans l'enceinte même des terreiros : un chef de culte de ma connaissanc à Belém, un jour où je

22. Voir MAGgIE (1977) pour la ville de Rio de Janeiro et BoYer-Araújo (1993a, 1993b) pour Belém.

23. En particulier Patricia Brrman (1980), Beatriz Dantas (1982) et Peter Fry (1984). L'institution judiciaire, souvent sollicitée pendant la période de répression des cultes, a également contribué au processus de classification et de hiérarchisation des pratiques et des croyances (MAGGIE 1988).

24. Certains chefs de culte serviront d'informateurs à des générations d'anthropologues : Martiniano do Bonfim, par exemple, est ainsi sollicité par Nina Rodrigues, Edison Cameiro, Arthur Ramos, Donald Pierson, Ruth Landes, E. Franklin Frazier (OliveIRA \& LiMA 1987).

25. J'ai mentionné plus haut son travail sur les lieux de culte de Rio de Janeiro publié pour la première fois en 1975. 
lui demandais de me préciser les différences entre umbanda et candomblé, sortit d'un tiroir un énorme registre et, pour toute réponse, me lut pendant plus d'un quart d'heure ce qu'il avait écrit sur un tout autre thème : les droits et (surtout) les devoirs des médiums.

Le statut social des érudits est, sans nul doute, un facteur qui explique la «valeur » différente attachée à leurs livres et à ceux des écrivains afro-brésiliens. La littérature ésotérique, rédigée et lue par des gens qui partagent une même appartenance de classe, jouit d'un prestige moindre qu'une œuvre scientifique produite par des représentants des classes dominantes, d'ordinaire plutôt enclines à conjuguer cultes de possession et superstition.

La seule distance sociale n'est toutefois pas suffisante pour comprendre l'influence des intellectuels sur les terreiros. Les lieux de culte ont depuis toujours été fréquentés par des membres des élites admettant aisément que des forces magico-religieuses puissent être manipulées et demandant aux pères et mères-de-saint de «travailler» en leur faveur 26.

\section{Tradition et syncrétisme, écriture et oralité}

Le point fondamental sur lequel les savants se différencient des proches des cultes, qu'ils soient médiums ou consultants, est qu'ils ne sollicitent pas (toujours) des services. Leur propos étant l' « étude», ils prennent une distance par rapport à l'événement et s'interrogent sur les catégories pertinentes du système quand les clients s'inquiètent de l'efficacité des pratiques. La distinction opérée par Michel de Certeau (1989: 85-86) entre tactique et stratégie nous permet d'appréhender les implications de la position particulière des anthropologues et de saisir les conséquences de leur travail sur la configuration présente de l'univers des cultes de possession: «J'appelle stratégie le calcul (ou la manipulation) des rapports de force qui devient possible à partir du moment où un sujet de vouloir et de pouvoir (une entreprise, une armée, une cité, une institution scientifique) est isolable [...] J'appelle tactique l'action calculée que détermine l'absence d'un propre. Alors aucune délimitation de l'extériorité ne lui fournit la condition d'une autonomie. »

C'est à l'évidence parce qu'ils parlent de ce « lieu du pouvoir et du vouloir propres » qu'est l'université que la compétence des savants est pleinement reconnue par l'ensemble de la société. La connaissance qu'ils sont censés produire n'est pas une connaissance de l'intérieur mais un savoir fondé sur la ratio"nalité scientifique qui lui donne ses critères de vérité. La référence à une tradition en somme rigidifiée peut instaurer une différenciation de l'espace religieux

26. Voir João Do Rıo (1976: 36), un journaliste qui fit une série de reportages sur les religions à Rio au début du siècle : «Les personnes éminentes ne manquent donc pas d'aller les entendre dans leurs bicoques, parce que les sorciers qui peuvent donner richesse, palais et éternité, qui changent à distance, avec une simple mixture de sang et d'herbes, l'existence humaine, habitent de pauvres maisons sordides, d'où émane une odeur nauséabonde. » 
dans la mesure où, en se construisant ailleurs, elle se soustrait aux règles qui président habituellement à l'évaluation des possédés.

On ne peut donc comprendre le peu d'effet sur les cultes de possession de cet effort d'écriture persistant des proches des terreiros qu'en considérant la logique spécifique mise en œuvre dans le traitement de la pensée. Dans la production scientifique, l'analyse permet d'organiser les données, chaque étape du raisonnement est formellement argumentée, les autres interprétations sont discutées et écartées quand elles semblent inadéquates. L'énumération caractérise au contraire la littérature ésotérique ; le degré de rigueur requis dans l'articulation des éléments du discours est faible et le texte, qui tient du catalogue de formules, trouve sa cohérence ailleurs qu'en lui-même 27.

"Écrire relève encore, pour une part, de l'ordre de l'oralité » (Zumthor $1987: 114)^{28}$. Cette remarque à propos de la littérature médiévale s'applique à la perfection aux ouvrages ésotériques. Chacun des livres consigne avant tout, en la transposant dans une forme écrite, une expression orale : l'agencement des parties, les idées exposées et le choix des mots sont, si l'on en croit leurs auteurs, dictés par les esprits. La prémisse d'une inspiration par les êtres invisibles est aussi un principe d'ouverture qui « esquive l'interprétation » (ibid. : 319 ) et la confrontation 29 : les révélations qui se poursuivent sans relâche interdisent que l'un des littérateurs prétende accéder à la totalité, à la vérité des cultes.

Mais les esprits n'ont d'autre matérialité que le corps des fils-de-saint qu'ils habitent. C'est donc à un contexte rituel où seules comptent la parole et la gestuelle que renvoient ces écrits. Or les affinités que se reconnaît un consultant avec un caboclo représenté par un médium et non par tel autre, ou sa préférence pour un orixá incarné dans un chef de culte plutôt que dans l'un de ses voisins, n'entraînent pas nécessairement un jugement quant à la réalité de la possession de celui qu'il aime moins. Les variations observables relèvent ici du «don » des fils-de-saint, de ce que les divinités consentent à leur donner. La compétence est irrévocablement liée à la performance sans cesse renouvelée.

Les caractéristiques de la littérature ésotérique sont à la fois sa force, puisque tout un chacun est en droit de consigner son interprétation du culte sans se prononcer directement sur l'opinion des autres, et sa faiblesse, parce que ce faisant aucun ne peut prétendre exprimer le point de vue de tous. Cette écriture imprégnée de marques orales ne peut produire de la norme; elle se trouve plus proche de la pure variation que de la pure répétition (Goody 1979: 209).

27. Le travail d'analyse de ces textes qui reste à faire sera entrepris ultérieurement.

28. Les quelques commentaires qui suivent doivent beaucoup au livre de Paul ZuMTHOR (1987), La lettre et la voix. Les réflexions de l'auteur sur l'influence réciproque de l'oralité et de l'écrit dans la constitution de la littérature médiévale ont ici été essentielles pour tenter de décrire et de comprendre la place que les gens des cultes accordent aux livres et l'usage qu'ils en font.

29. Les termes de l'analyse de Jack Goopy $(1979: 208)$, à propos de l'Invocation au Bagré dont il existe plusieurs versions écrites, conviennent également à cette littérature : «Certains de ces écarts [par rapport au modèle] pouvaient éventuellement être reconnus par les locuteurs comme des 'fautes '. Cependant [...] chaque individu possède son propre modèle de référence, parce qu'il n'y a aucun moyen de correction automatique ni aucun système bien établi de régulation autoritaire.» 
C'est au point précis de l'écart entre acte scientifique et acte magique, tel que les décrit Tzvetan Todorov (1978: 279), de leurs objectifs distincts et de leurs logiques différentes, que la production savante est instituée comme référence : «L'acte scientifique est d'abord un acte qui se veut purement référentiel [...] ; de surcroît, le savant n'admet pas qu'il cherche, en son activité scientifique même, à transformer la réalité qu'il décrit. On ne peut assimiler la magie à la science qu'en réduisant l'énonciation au pur énoncé, qu'en gommant la nature performative de la magie. »

La tradition devient l'objet de la conquête des chefs de culte dans une concurrence médiatisée par les scientifiques. La référence africaine, qui modère sensiblement la perception négative qu'ont les élites des cultes de possession, intervient en effet dans la définition des positions respectives des maîtres de terreiro entre eux et face à la société environnante ${ }^{30}$. Les pères et mères-desaint accèdent par leur truchement à de nouveaux moyens de légitimation et puisent dans la littérature un langage dans lequel exprimer leur rivalité : de la conformité à «ce qui est écrit dans les livres » dépend la reconnaissance de l'autorité religieuse et de l'existence sociale des chefs de culte ${ }^{31}$. Les pratiques rituelles (les étapes de l'initiation, le jeu des cauris, la représentation des orixás, etc.) peuvent idéalement être confrontées à un corpus écrit qui en définit le cadre. De ce fait, ce n'est plus une forme des cultes (le candomblé) parmi d'autres qui est exportée dans d'autres régions du pays par des maîtres de terreiro migrants mais une «tradition» soutenue par des textes. Les érudits ont ainsi favorisé la constitution d'une « tradition » pensée comme orale mais qui, par sa rigidité, porte la marque du savoir livresque qui la fonde.

La participation des ethnologue en tant que membres à part entière des terreiros modifie pourtant le statut de leurs écrits 32 . Leur compétence sur le thème des cultes, qui dérivait de l'extériorité du lieu de production des ouvrages scientifiques, tend à se confondre avec leur qualification religieuse, à savoir les initiations qu'ils ont suivies. L'expérience dans les cultes appuie alors le discours de la science. Ce mouvement est inverse de celui qu'esquissent les proches des terreiros quand ils tentent de prouver leur maîtrise de l'écrit, symbole de l'instruction, en « faisant des livres » imprimés ou manuscrits, plus simplement en montrant leur connaissance des grandes lignes exposées par les

30. Sur le processus de définition des positions dans l'univers religieux, voir Michel AGIER (1992: 7475) à propos de la société bahianaise : « Pour produire cette identité politique, les diverses formes qui institutionnalisent la culture " nègre" tentent de contrôler les pratiques. Non pas en empêchant l'émergence des formes nouvelles [...], mais en leur donnant un sens, c'est-à-dire en les classant et en les hiérarchisant. "

31. Un exemple évident de la reconnaissance par les élites de certains maîtres de terreiro est la décision prise par le maire de Bahia de décréter trois jours de deuil officiel à la mort, en août 1986, de Mäe Menininha, mère-de-saint de l'un des «plus » traditionnels terreiros de la ville. À Brasilia aussi, les sénateurs, pendant leur session, ne manquèrent pas d'exprimer leurs regrets.

32. Des chercheurs ont choisi de participer de très près aux cultes : Pierre Verger, « détenteur d'une haute charge " (AUGRas 1983: 51) dans un candomblé de Bahia, soutient le père Balbino ; l'anthropologue d'origine argentine Juana Elbein dos Santos, épouse d'un important personnage des cultes, Maître Didi, « se consacre à la systématisation de la vision nagô " (ibid. : 52); l'ethnologue Gisèle Cossard est quant à elle mère-de-saint à Rio de Janeiro... 
anthropologues dans leurs ouvrages. Dans le premier cas, la distance à l'objet se réduit progressivement jusqu'à disparaître ; dans l'autre, l'implication personnelle précède la tentative de systématisation.

De cette participation des intellectuels à l'univers des cultes les maîtres de terreiro s'autorisent pour disposer de la littérature scientifique comme ils le font de la littérature ésotérique, «à la mode du don » et de l'inspiration. Car si nul dans le milieu des cultes n'ignore ce qui sépare le candomblé de l'umbanda, nul à l'inverse ne méconnaît ce qui les rapproche et ne se prive de les accommoder. Et ce avec d'autant plus d'aisance que dans l'initiation, le « secret », que n'ont pas dévoilé les ouvrages anthropologiques, est un espace libre où la rigueur de la réplique n'est pas contrôlée et où l'innovation peut être introduite 33 .

Les commérages dans le milieu des cultes, qui dénoncent toujours une infraction aux énoncés de la « tradition », n'épargnent en conséquence pas plus un père-de-saint qui a retenu l'attention d'un anthropologue et reçoit son soutien que les autres.

CNRS

CERMACA, EHESS, 54 bd Raspail, 75006 Paris

MOTS CLÉS : Brésil - culte de possession — écriture - oralité — tradition

\section{B I B L I O G R A P H IE}

AGrer, Michel

1992 «Ethnopolitique : racisme, statuts et mouvement noir à Bahia ». Cahiers d'Etudes africaines $125: 53-81$.

Augras, Monique

1983 O duplo e a metamorfose. Petrópolis, Vozes.

Barreto Ramos, Daisy

1994 «Plasticité et résistance : le mythe et le culte de Maria Lionza au Venezuela », Gradhiva 15 : 81-88.

Birman, Patricia

1980 Feitiço, carrego e olho grande. Os males do Brasil são. Rio de Janeiro, Museu Nacional, Dissertação de Mestrado.

BOUGEror, Christiane

1993 «Le cumul magico-religieux à la Guadeloupe », Journal de la Société des Américanistes 79 : 91-103.

Boyer-Araújo, Véronique

1993a Femmes et cultes de possession: les compagnons invisibles. Paris, L'Harmattan.

33. L'opposition entre don et initiation n'est pas sans évoquer par exemple le conflit entre la lettre et l'esprit dans le mouvement Aladura au Nigeria (Probst 1989) où les prophètes répliquent aux pasteurs que « l'écrit n'est pas le seul chemin vers la vérité ». Dans les cultes de possession brésiliens, cette idée est parfois renforcée par un discrédit jeté sur le savoir livresque ou une vigoureuse déclaration d'analphabétisme de la part des médiums. 
$1993 \mathrm{~b}$ «Les ' traditions' risquent-elles d'être contaminées ? Paradigmes scientifiques et orthodoxie religieuse dans les cultes de possession au Brésil », Journal de la Société des Américanistes $79: 67-90$.

Brown, Diana

1974 Umbanda : Politics of an Urban Movement, Ph. D. Dissertation, Columbia University, Department of Anthropology.

Carneiro, Edison

1964 Ladinos e crioulos. Rio de Janeiro, Ed. Civilizaçåo Brasileira.

Certeau, Michel DE

1989 L'invention du quotidien, 1. Arts de faire. Paris, 10/18.

DanTas, Beatriz Gois

1982 « Repensando a pureza nagô », Religiäo e Sociedade, 8 juillet : 15-20. Rio de Janeiro.

FRY, Peter

1984 « De um observador não participantes », Communicaçöes do ISER, 8 : 37-46. Rio de Janeiro.

GooDY, Jack

1979 La raison graphique. Paris, Éditions de Minuit.

MaGgre, Yvonne

1977 Guerra de Orixá : um estudo de ritual e conflito. Rio de Janeiro, Zahar editores.

1988 O medo do feitiço : relações entre magia e poder no Brasil. Thèse de doctorat, Rio de Janeiro, Museu Nacional.

Oliveira, Waldir Freitas \& Vivaldo Costa Lima

1987 Cartas de Edison Carneiro a Arthur Ramos, São Paulo, Corrupio.

ORTIZ, Renato

1978 A morte branca do feiticeiro negro. Petrópolis, Vozes.

Prandi, Reginaldo

1991 Os Candomblés de São Paulo. São Paulo, Editora Hucitec.

Probst, Peter

1989 « The Letter and the Spirit : Literacy and Religious Authority in the History of the Aladura Movement in Western Nigeria ", Africa 59 (4) : 478-495.

Rro, João Do

1976 As religiōes no Rio. Rio de Janeiro, Ed. Nova Aguilar.

ToDorov, Tzvetan

1978 Les genres du discours. Paris, Le Seuil.

VERGolino, Anaíza

1976 O tambor das flores : uma análise da federação espírita umbandista e dos cultos afrobrasileiros do Pará (1965-1975). Campinas, Dissertação de Mestrado, UNICAMP.

ZEMPLÉNI, Andras

1976 «La chaîne du secret », Nouvelle Revue de Psychanalyse 14 : 313-324.

ZuMTHOR, Paul

1987 La lettre et la voix. Paris, Le Seuil.

\section{LITTÉRATURE UMBANDISTE}

Collectif

1960 Umbanda, religião do Brasil. Săo Paulo, Editora Obelisco.

Freitas, B. T. \& T. da Silva Pinto

1970 Doutrina e ritual de umbanda. Rio de Janeiro, Editora Espiritualista.

Matta e Silva, W. W. DA

1967 Doutrina secreta da umbanda. Rio de Janeiro, Livraria Freitas Bastos. 
SALES, Nívio Ramos

1981 Prova de fogo : posando para retrato. Rio de Janeiro, Esquina Editora.

Trindade, Diamantino F.

1991 Umbanda e sua historia. São Paulo, Icone Editora.

\begin{abstract}
Veronique Boyer, The Gift and Initiation: About the Impact of Literature on Cults of Possession in Brazil-The mediums of Brazilian possession cults contrast gifts with initiation. This contrast can bee understood by studying how scientific and esoteric writings have shaped this religious field. Writings based on scientific paradigms have defined ritual norms that the initiatory system supposedly maintains. Having failed in their attempst at systematization, esoteric writings have encouraged personal interpretations based on inspiration by the spirits. The forming of a religious «tradition » can be understood only by taking into account the ambivalent relations between speech and writing.
\end{abstract}

\title{
RESUMEN
}

Véronique BoYer, El don y la iniciacíon. Del impacto de la literatura sobre los cultos de posesión en Brasil. - En Brasil, los mediums oponen el don y la iniciación durante los cultos de posesión. Considerando las incidencias de escritos, científicos y esotéricos, trataremos de comprender esta oposición en en la actual constitución de campo religioso. Los primeros, apoyados en los paradigmas del conocimiento erudito, han definido efectivamente las normas rituales que mantendria un sistema iniciatico. Los segundos, por el contrario, han fracasado en su empresa de sistematización, cuando incentivan la expresión de versiones personales inspiradas por los espíritus. La formación de una «tradición » religiosa solo puede ser considerada si tenemos presente las relaciones ambivalentes entre escritura y oralidad. 